\title{
Transpleural systemic artery to pulmonary vessel fistulas: Imaging characteristics and management of an uncommon entity
}

Thomas J An, T. Gregory Walker*

Department of Radiology, Division of Interventional Radiology, Massachusetts General Hospital, Boston, MA, USA

Received: August 30, 2020

DOI: $10.5430 / d c c . v 7 n 3 p 7$
Accepted: December 31, 2020

Online Published: January 3, 2021

URL: https://doi.org/10.5430/dcc.v7n3p7

\begin{abstract}
Systemic arterial to pulmonary vessel fistulas may develop in the setting of pleural inflammation and/or injury. Bronchial arteries are the most commonly involved vessels, with recruitment often occurring in chronic diseases such as tuberculosis and cystic fibrosis. Rarely, transpleural systemic arteries that directly communicate with the pulmonary vasculature may develop in the presence of pleural injury or chronic inflammation. The clinical presentation and management of three cases of transpleural systemic artery to pulmonary vessel fistulas are detailed. Given the atypical imaging appearance of these lesions, there is risk of misdiagnosis of these entities as a pulmonary arteriovenous malformation. Understanding the pathophysiology, complex anatomy, and treatment for these rare vascular malformations is crucial prior to angiographic intervention in order to improve outcomes, avoid misdiagnosis, and prevent inappropriate intervention.
\end{abstract}

Key Words: Pulmonary arteriovenous fistula, Transpleural, Pulmonary hemorrhage

\section{INTRODUCTION}

Systemic artery to pulmonary artery and vein fistulas may develop in the setting of chronic lung inflammation, with the inflammatory response leading to decreased perfusion, compensatory vascular hypertrophy, and collateral development. Bronchial arteries are typically the first systemic arteries involved, with recruitment commonly occurring in the setting of chronic pulmonary diseases such as tuberculosis and cystic fibrosis.

On rare occasions, transpleural systemic arteries that directly communicate with the pulmonary vasculature may develop in the setting of pleural inflammation and/or injury. Given the rarity of this diagnosis, these vascular malformations may be misdiagnosed as pulmonary arteriovenous malformations and result in inappropriate or ineffective intervention.

This report details the pathophysiology, diagnosis, and endovascular management of three patients from our institution with a history of pleural trauma or inflammation that developed transpleural fistulas between systemic arteries and the pulmonary arteries and veins.

\section{CASE PResentation}

\subsection{Patient 1}

A 57-year-old man with a history of video-assisted thoracoscopic surgical (VATS) resection of a right chest wall schwannoma presented with chest pain and dyspnea. A contrast

\footnotetext{
*Correspondence: T. Gregory Walker; Email: tgwalker@mgh.harvard.edu; Address: Massachusetts General Hospital, 55 Fruit Street, GRB 298, Boston, MA 02114, USA.
} 
enhanced CT demonstrated a vascular malformation in the periphery of the right middle and lower lobes. This finding was initially diagnosed as a pulmonary arteriovenous malformation. However, endovascular pulmonary angiography failed to demonstrate an abnormality.

Subsequent computed tomography angiography (CTA) demonstrated prominent right inferior phrenic, lateral thoracic, and internal mammary arteries supplying the malformation with outflow to pulmonary arteries and veins. He underwent a total of four staged transcatheter embolizations of the feeding arteries and nidus with coils and onyx (see Figure 1) with significant size reduction and stable appearance of the residual vascular malformation over 5 years.

\subsection{Patient 2}

A 24-year-old man with a history of right sided VATS wedge resection and talc pleurodesis as a teenager for a sponta-

neous pneumothorax presented with hemoptysis. A contrast enhanced CT demonstrated a right lower lobe vascular malformation. He underwent subsequent partial VATS resection of the malformation.

He presented two years later with recurrent hemoptysis and chest pain. Digital subtraction angiography demonstrated multiple hypertrophied right sided systemic arteries including the inferior phrenic, internal mammary, lateral thoracic, and intercostal arteries communicating with the pulmonary arteries and veins (see Figure 2). Repeat surgical resection was considered unwise given the risk of uncontrollable hemorrhage and presumed high likelihood of postoperative recurrence. He underwent a total of five staged transcatheter embolizations with onyx and ethanol with eventual reduction in size of the vascular malformation and resolution of hemoptysis.
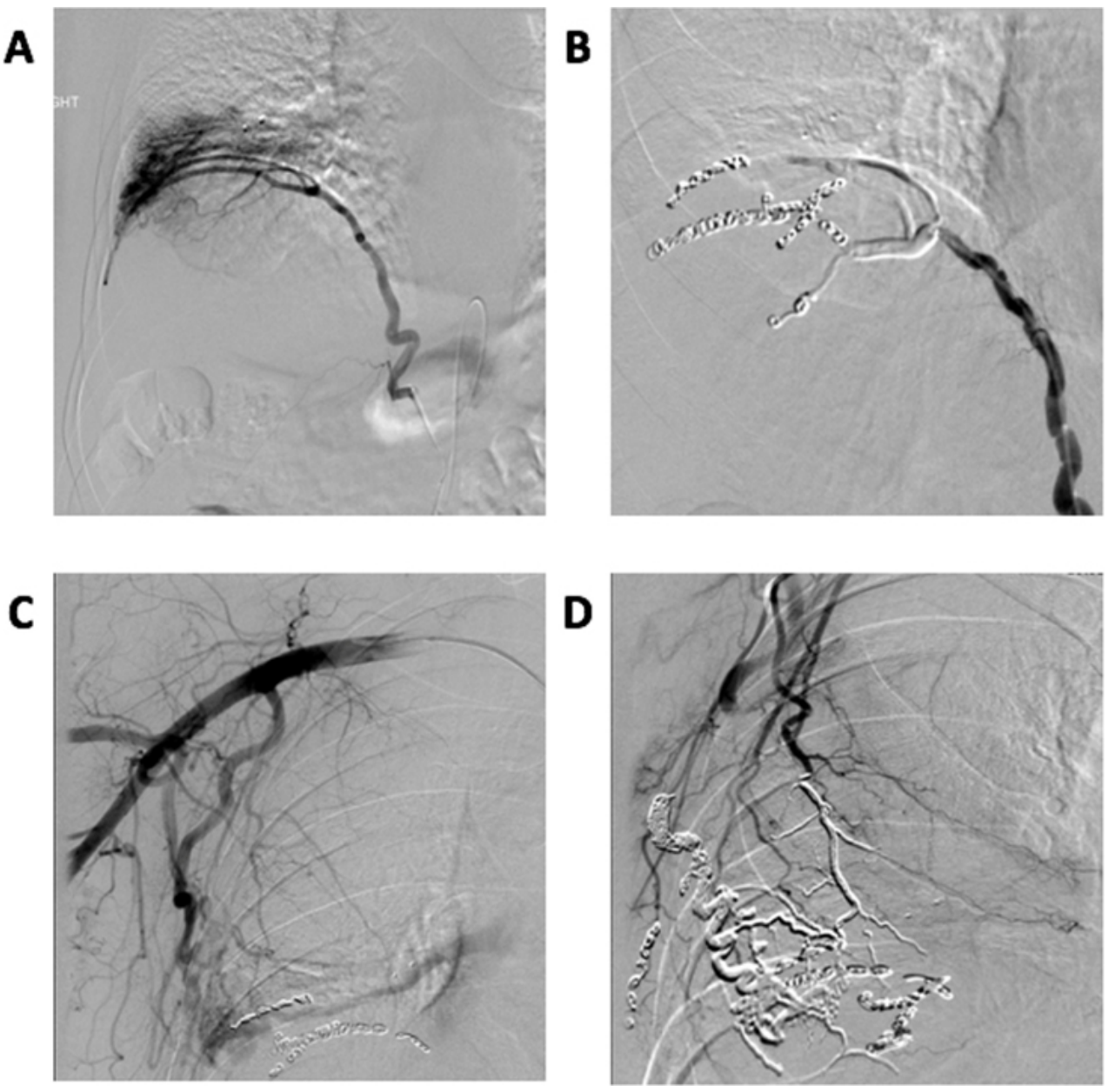

Figure 1. A. Angiogram of the right inferior phrenic artery demonstrates communication with a vascular malformation involving the pulmonary vessels; B. Post coil embolization of multiple branches from the inferior phrenic artery without evidence of filling of the vascular malformation; C. Angiogram of the right subclavian artery demonstrates additional communication between the lateral thoracic artery and the vascular malformation; D. Post coil and Onyx embolization of multiple supplying branches from the lateral thoracic artery 


\subsection{Patient 3}

A 42-year-old man with primary sclerosing cholangitis and cholangiocarcinoma underwent right hepatectomy complicated by a chronic right subphrenic biloma. He later developed a fistula to the skin and presented with blood clots passing from the orifice of the fistula. A CTA demonstrated multiple hypertrophied, serpiginous right subphrenic arteries overlying the hepatic dome coursing through the biloma.
Angiography demonstrated right inferior phrenic artery hypertrophy with abnormal transpleural shunting to the right lower lobe pulmonary arteries and veins (see Figure 3). The inferior phrenic artery was embolized with 700-900 $\mu \mathrm{m} \mathrm{Em-}$ bospheres without evidence of residual filling of the vascular malformation. The patient was discharged without any postprocedural complications or further clot passage.
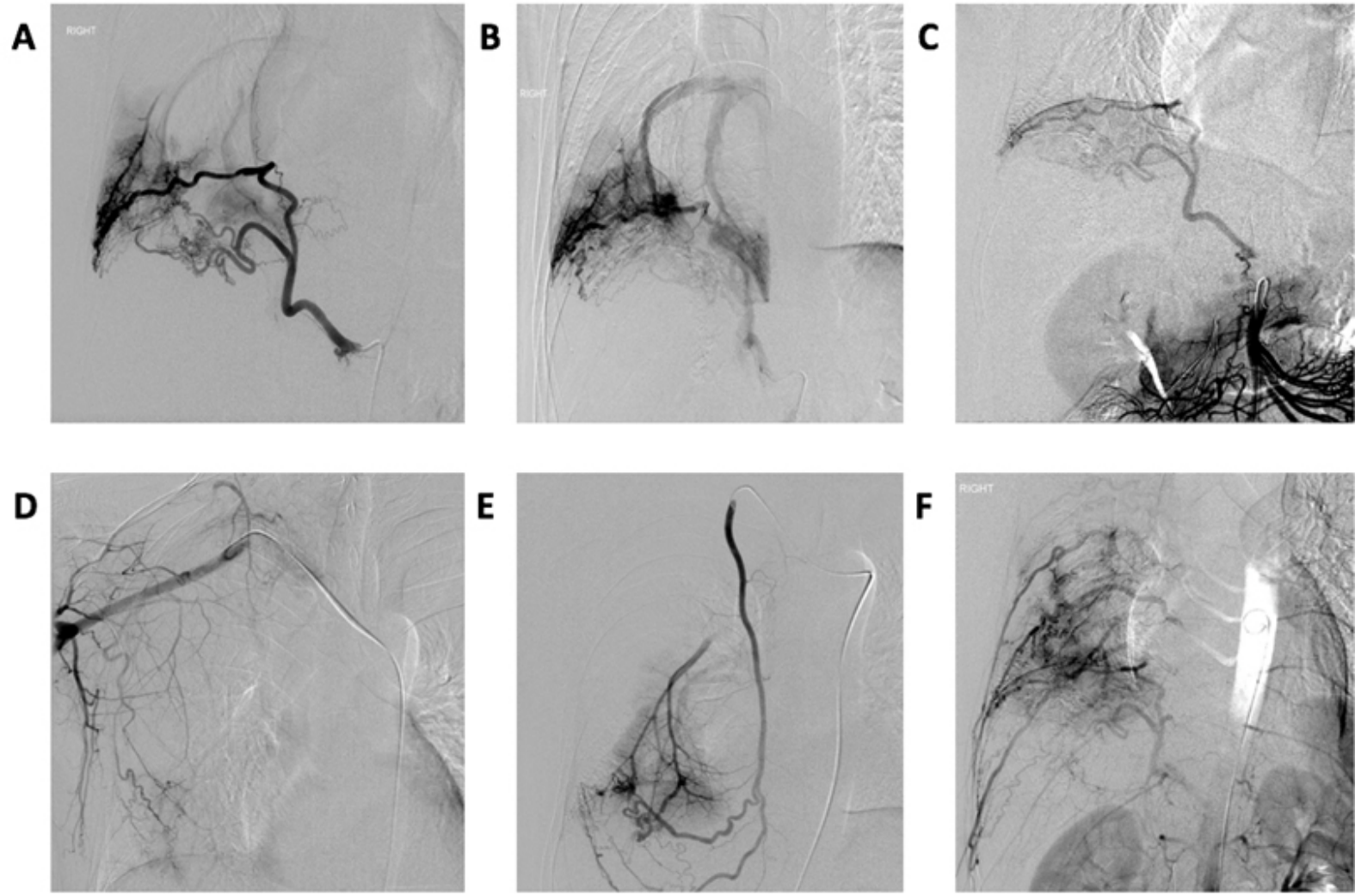

Figure 2. Angiogram demonstrating communication between the vascular malformation and the inferior phrenic artery (Aearly arterial phase and B - delayed phase with filling of the pulmonary veins), superior mesenteric artery (C), lateral thoracic artery (D), internal mammary artery (E), and multiple intercostal arteries (F)

\section{DISCUSSION}

The three patients described in this report had a history of pleural trauma or inflammation that resulted in rare acquired transpleural fistulas involving systemic arteries and the pulmonary vasculature.

Pulmonary recruitment of collateral systemic arteries is mediated by angiogenic molecular pathways in the setting of inflammation that causes local reduction of the pulmonary circulation. ${ }^{[1]}$ Although bronchial arteries are the most common systemic arteries to hypertrophy in the setting of chronic pulmonary inflammation, similar angiogenic pathways may lead to systemic transpleural vessel recruitment in the setting of pleural disease. ${ }^{[2]}$ Patients with transpleural systemic artery to pulmonary vessel fistulas typically present with symptoms including dyspnea, angina, or hemoptysis, with the severity dependent on the fistula size and complexity.

Hypertrophy of systemic arteries with fistulization to pulmonary arteries and veins has previously been described. ${ }^{[3]}$ A review of 80 case reports of fistulas between the internal mammary artery and the pulmonary vasculature noted that coronary artery bypass surgery was the etiology for fistula in the majority of cases. ${ }^{[4]}$ However, transpleural systemic arterial to pulmonary vessel fistulas arising from abdominal arteries such as the inferior phrenic artery, as described in this case series, are rare. ${ }^{[5-9]}$

These entities are prone to misdiagnosis if the imaging study used to characterize the lesion is not optimized for evaluation 
of the systemic arteries involved. For example, a systemic to pulmonary vessel fistula may be misdiagnosed as typical pulmonary arteriovenous malformations if a contrast enhanced CT examination fails to opacify the systemic arteries involved. Misdiagnosis as a pulmonary embolism on pulmonary angiogram CT may also occur if pulmonary arterial filling defects from unenhanced blood delivered into the pulmonary arteries by the systemic arteries are present. ${ }^{[9]}$ Pre-procedural CTA is crucial for effectively identifying transpleural systemic arterial contributions to a fistula. A pleural thickness of more than $3 \mathrm{~mm}$ and enhancing vascular structures within the extra-pleural fat layer are suggestive of a transpleural systemic arterial supply to a fistula. ${ }^{[10]}$
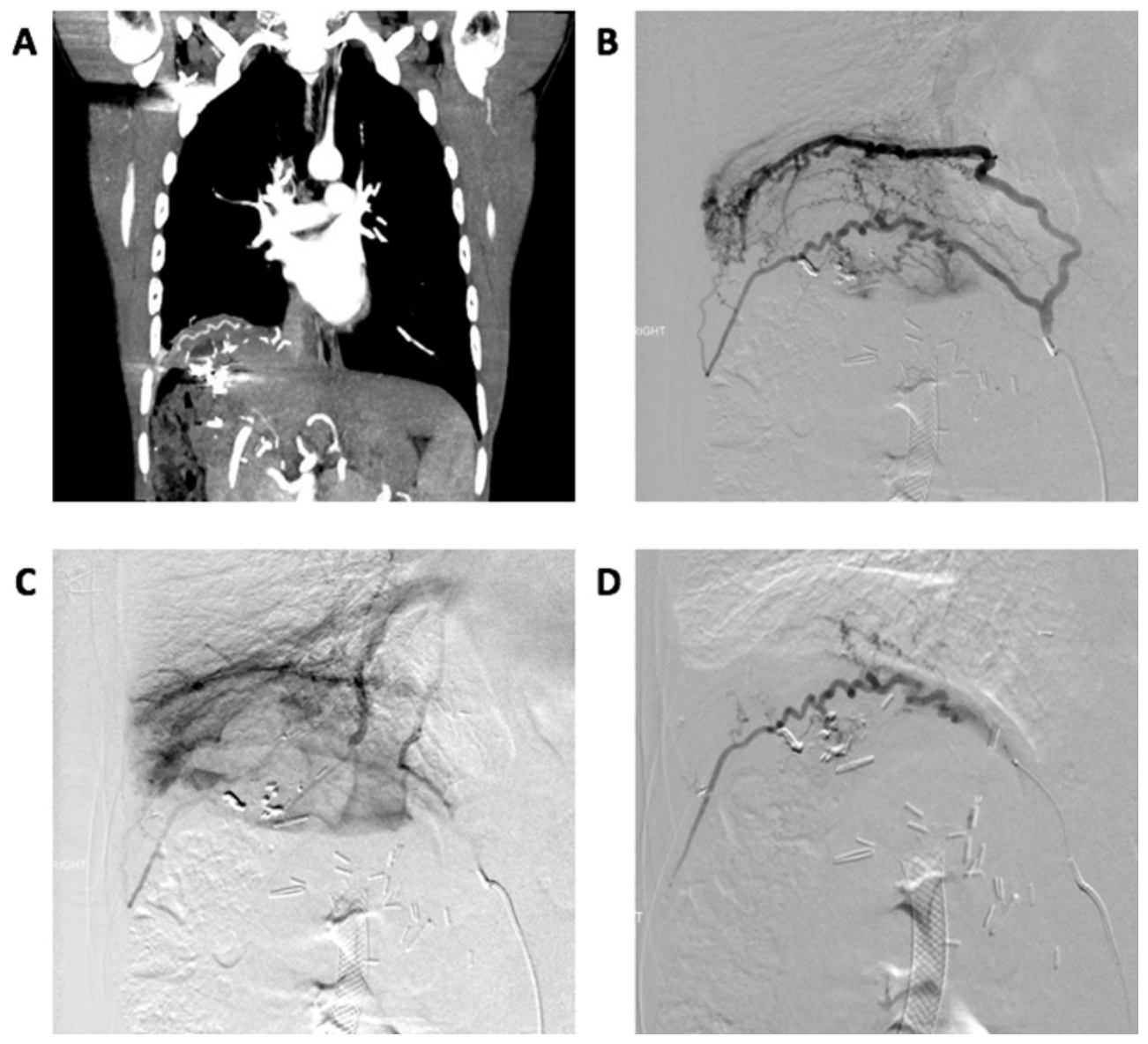

Figure 3. A. Coronal maximal intensity projection image demonstrates a right subphrenic hypoattenuating collection with a prominent right inferior phrenic artery; B. Angiogram of the right inferior phrenic artery demonstrates a communication with the pulmonary vasculature; C. Delayed image acquisition demonstrates filling of the pulmonary veins; D. Post embolization with 700-900 $\mu \mathrm{m}$ Embospheres without residual filling of the vascular malformation

Although there is no standard treatment algorithm for these fistulas, embolization is favored due to the high morbidity associated with surgery and a presumed high post-operative recurrence rate. In prior case reports of systemic to pulmonary artery fistulas involving the inferior phrenic artery, treatments varied between expectant management, embolization only, and embolization with surgical resection. ${ }^{[5-9]}$ In our series, all patients were managed with single or staged embolization, although one had undergone surgical resection prior to embolotherapy. Embolic agents ranged between coils, liquid embolics (e.g., Onyx), and beads, depending of operator preference. Post-embolization CT demonstrated some degree of persistence of the systemic to pulmonary artery fistula in two of the three cases in this case series. However, in the absence of further symptoms, these vascular malformations may be monitored with serial CT examinations with the option for additional embolization procedures as needed. Systemic artery to pulmonary vessel fistulas also have the potential to affect the hemodynamic parameters of the pulmonary circulation and alter pulmonary artery pressures or pulmonary vascular resistance. Unfortunately, due to the retrospective nature of this case series, pulmonary hemodynamic parameters were not obtained at the time of endovascular intervention. 
Given the rarity of transpleural systemic artery to pulmonary vessel fistulas, these vascular malformations are prone to misdiagnosis and mistreatment. The presence of a pulmonary vascular malformation in the setting of prior pleural injury or inflammation should raise suspicion for potential transpleural systemic artery to pulmonary vessel fistula. Accurate diagnosis and characterization of this rare entity with CT angiography is essential prior to attempting embolotherapy to avoid non-therapeutic interventions.

\section{Conflicts of InTEREST Disclosure}

The authors declare they have no conflicts of interest.

\section{REFERENCES}

[1] McDonald D. Angiogenesis and Remodeling of Airway Vasculature in Chronic Inflammation. Am J Respir Crit Care Med. 2001; 164: S39-S45. PMid:11734465. https ://doi.org/10.1164/ajrccm .164.supplement_2.2106065

[2] Chun JY, Morgan R, Belli AM. Radiological Management of Hemoptysis: A Comprehensive Review of Diagnostic Imaging and Bronchial Arterial Embolization. Cardiovasc Intervent Radiol. 2010; 33(2): 240-250. PMid:20058006. https ://doi.org/10.1007/s00270 $-009-9788-z$

[3] Noë GD, Jaffé SM, Molan MP. CT and CT angiography in massive haemoptysis with emphasis on pre-embolization assessment. Clin Radiol. 2011; 66(9): 869-875. PMid:21658690. https://doi .or g/10.1016/j.crad. 2011.03.001

[4] Abdul Jabbar A, Patel A, Marzlin N, et al. Internal mammary arteryto-pulmonary vasculature fistula: Systematic review of case reports. Vasc Med. 2017; 22(5): 426-431. PMid:28990495. https: //doi.org/10.1177/1358863X17724262

[5] Webb W, Jacobs R. Transpleural abdominal systemic arterypulmonary artery anastomosis in patients with chronic pulmonary infection. Am J Roentgenol. 1977; 129(2): 233-236. PMid:409155. https://doi.org/10.2214/ajr.129.2.233
[6] Yakushiji E, Ota S, Komatsu T, et al. Massive Hemoptysis due to Right Inferior Phrenic Artery-to-Right Pulmonary Artery Fistula in the Right Middle Lobe of the Lung. Intern Med. 2017; 56(6): $687-$ 689. PMid:28321071. https://doi.org/10.2169/internalme dicine.56.6783

[7] Hsu SJ, Luo YH, Lee YC, et al. Life-threatening hemoptysis due to left inferior phrenic artery to pulmonary artery fistula rescued by extracorporeal membrane oxygenation therapy. Interact Cardiovasc Thorac Surg. 2011; 12(2): 337-338. PMid:21097453. https : //doi.org/10.1510/icvts.2010.255265

[8] Denlinger CE, Egan TM, Jones DR. Acquired systemic-to-pulmonary arteriovenous malformation secondary to Mycobacterium tuberculosis empyema. Ann Thorac Surg. 2002; 74(4): 1229-1231. https : //doi.org/10.1016/S0003-4975(02)03860-2

[9] Alsafi A, Shovlin CL, Jackson JE. Acquired Transpleural Systemic Artery-to-Pulmonary Artery Communication Mimicking a Pulmonary Arteriovenous Malformation and Causing a False-Positive Diagnosis of a Pulmonary Embolus. J Vasc Interv Radiol. 2018; 29(9): 1313-1315. PMid:30146210. https://doi.org/10.1016/ j.jvir.2017.12.016

[10] Yoon W, Kim YH, Kim JK, et al. Massive Hemoptysis: Prediction of Nonbronchial Systemic Arterial Supply with Chest CT. Radiology. 2003; 227(1): 232-238. PMid:12601194. https ://doi.org/10.1 $148 /$ radiol. 2271020324 